\title{
A Novel Palmprint Recognition System based on GIST and ELM
}

\author{
Dr. S. Chidambaranathan
}

Head, Department of Computer Applications, St. Xavier's College (Autonomous), Tirunelveli, India

\begin{abstract}
The biometric security systems are at limelight recently. Palmprint recognition is a strong security solution and has gained substantial interest. This paper aims at recognizing the palmprint by utilizing several features and Extreme Learning Machine (ELM) is employed as the classifier. The palmprint images are acquired and preprocessed. The preprocessing phase converts the original palmprint image into binary format. The feature extraction is the next significant phase of this work and this is accomplished by GIST descriptors. The final step is the classification, which aims at effective recognition of palmprints. This work employs ELM as the classifier. The experimental outcome of the proposed work is satisfactory and is evident through the results.
\end{abstract}

Keywords: palmprint recognition, GIST, ELM

\section{Introduction}

The biometric security systems are at limelight recently. Palmprint recognition is a strong security solution and has gained substantial interest [1-5]. Palmprints provide several unique information which paves way for effective recognition of an individual. Palmprints are more powerful than fingerprints, owing to several unique features such as ridges, valleys, minutiae, principal lines, wrinkles and pores. The pattern area of palmprint is quite larger, which makes it possible to have numerous features.

The palmprint recognition systems are classified into two categories. They are low-resolution features based techniques and high resolution features based techniques. The lowresolution features based techniques consider the principal lines, wrinkles and textures. The existing techniques of this subclass employ edge detection methods for palm lines extraction. Certain techniques extract specific features initially, which is followed by the subspace projection. This is to reduce the dimensionality and the reduced features are compared by classifiers.

The high resolution features based techniques exploit high resolution images. Thus, multiple unique features such as ridges, singular points and minutiae can be extracted from the images. However, extracting feature from palmprints is a major issue. The reasons are the quality of palmprints to be recognized and the vast area of palm.

This paper aims at recognizing the palmprint by utilizing several features and Extreme Learning Machine (ELM) is employed as the classifier. The proposed work employs GIST descriptor for the feature extraction process. Thus, the recognition accuracy of the proposed work is satisfactory and is shown in the experimental analysis section.

The remainder of this paper is organized as follows. Section 2 presents the review of literature. The proposed approach is presented in section 3. Section 4 is loaded with the experimental analysis. The concluding remarks are given in section 5 .

\section{Review of Literature}

In Jain and Feng [2009], the discriminative palmprint features are grouped into two subclasses: ridges and creases. In Jain et al. [2007], ridge features are further divided into three levels: Level 1 (ridge pattern), Level 2 (minutia points), and Level 3 (pores and ridge contours). Level 1 and Level 2 features can be extracted from fingerprint or palmprint images with 500 or less dpi, while Level 3 features should be extracted from 1000 dpi images. For ridge-based palmprint recognition, various Level 1 and Level 2 feature extraction and matching techniques developed for fingerprint recognition can be directly adopted. Minutiae extraction and descriptor are also proposed by taking the characteristics of latent palmprint into account [Jain and Demirkus 2008; Jain and Feng 2009].

Recently, the discriminative power of Level 3 features has been noticed by the biometric and forensics communities. Jain et al. [2007] have developed a hierarchical matching system to utilize Level 3 features for performance improvement in fingerprint matching. Although the acquisition and processing time of the high resolution palmprint images may restrict its applications in online personal authentication systems, ridge features are crucial for latent palmprint recognition, which has shown great potential in forensics and law enforcement. Creases, also referred to as palm lines, include principal lines and wrinkles, which are obvious structural human features adopted for use in palmprint identification.

The principal lines and some main wrinkles are formed several months after conception, and the other wrinkles are formed as the consequence of both genetic effects and various postnatal factors. The complex patterns of creases carry rich information for personal authentication. Most creases could be acquired with a low resolution scanner (100 dpi). Subsequently, online capture devices were developed to collect low resolution palmprint images in real time. Nowadays, low resolution palmprint recognition has gradually become a focus of research interest in the field of palmprint recognition [Li et al. 2002; Zhang et al. 2003]. 


\section{International Journal of Science and Research (IJSR) \\ ISSN (Online): 2319-7064}

Index Copernicus Value (2013): 6.14 | Impact Factor (2014): 5.611

In order to develop effective feature representation approaches, rather than explicitly extracting palm lines, low resolution palmprint images can also be treated as texture images, and texture descriptors are then used to describe invariant palmprint features. With the rapid progress in sensor techniques and increase of computational power, except for low resolution palmprint recognition, several novel palmprint recognition technologies have been recently developed.

Motivated by the above works, the proposed work presents a promising palmprint recognition system which relies on GIST descriptor and ELM classifier.

\section{Proposed Approach}

The proposed work is compartmentalized into palmprint image acquisition, pre-processing, feature extraction and classification. All these stages are explained in this section.

\subsection{Palmprint Image Acquisition}

The palmprints can be acquired either by online and offline. The online palmprint acquisition approach exploits CCDbased palmprint scanners. This approach captures high quality images with proper alignment. On the other hand, the offline palmprint image acquisition method scans every palm by the application of ink. However, this method is not practically possible. Several online datasets are available for palmprints, which can be exploited without any hassles.

\subsection{Pre-processing}

Pre-processing is the fundamental operation which processes the images, in order to make the images suitable for the succeeding processes. As an initial step, the pre-processing stage converts the original palmprint images into binary images. The key-points are then detected and the region of interest is extracted.

\subsection{Feature Extraction}

The GIST descriptor has recently received increasing attention in the context of scene recognition. In this paper we evaluate the search accuracy and complexity of the global GIST descriptor for two applications, for which a local description is usually preferred: same location/object recognition and copy detection.

First, a grayscale image is pre-processed by a whitening filter to preserve dominant structural details and then normalized with respect to local contrast. The pre-processed image is then passed through a cascade of Gabor filter in $\mathrm{S}$ scales with $\mathrm{O}$ orientations at each scale. Each of these $\mathrm{S} \times \mathrm{O}$ images (orientation maps), representing the original image at one orientation in each scale, is then divided into an N-by-N grid. Within each block on the grid, the average intensity is calculated to represent the feature in that block. The final output is a concatenated feature vector of $\mathrm{S} \times \mathrm{O} \times \mathrm{N} \times \mathrm{N}$ dimensions.
The GIST descriptor has proved to be powerful in scene categorization and retrieval. Each image is convolved with Gabor filter at 8 scales and 8 orientations. The filter responses are averaged within each of $4 \times 4$ divisions of the image.

\subsection{Classification}

This work employs Extreme Learning Machine as classifier, so as to recognize the palmprints. The reasons for incorporating ELM are as follows. ELM was originally developed from feedforward neural networks. With this extension, the unified ELM solution can be obtained for feedforward neural networks, RBF network, LS-SVM, and PSVM.

Furthermore, ELM, which is with higher scalability and less computational complexity, not only unifies different popular learning algorithms but also provides a unified solution to different practical applications (e.g., regression, binary, and multiclass classifications). Different variants of LS-SVM and SVM are required for different types of applications.

ELM avoids such trivial and tedious situations faced by LSSVM and SVM. In ELM method, all these applications can be resolved in one formula. From the optimization method point of view, ELM and LS-SVM have the same optimization cost function; however, ELM has milder optimization constraints compared to LS-SVM and PSVM. As verified by simulations, the resultant ELM method can run much faster than LS-SVM. ELM with random hidden nodes can run even up to tens of thousands times faster than SVM and LS-SVM.

\subsection{Overall algorithm of the proposed work}

The overall algorithm of the proposed work is given below.

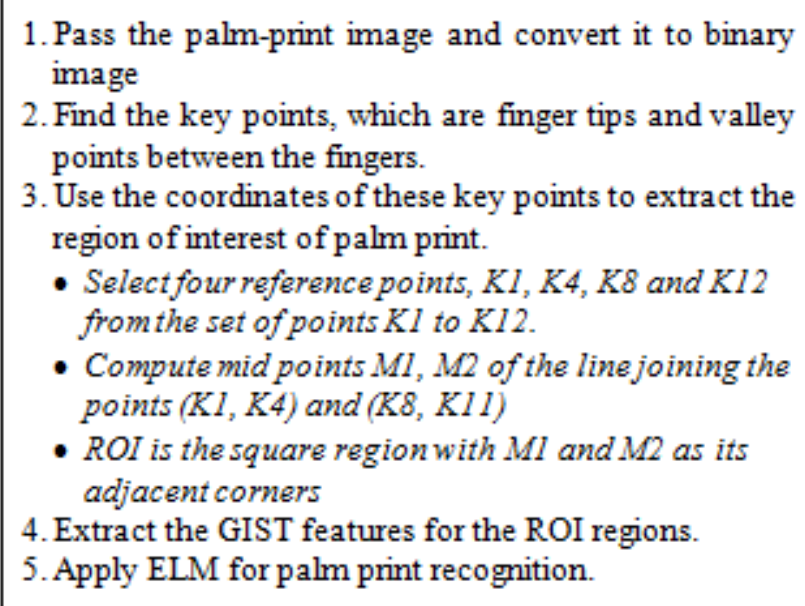

Initially, the palmprint images are converted to binary format. Then, with reference to the finger tips and valley points between the fingers, the key points are found out. The coordinated of these keypoints are utilised to extract the ROI of the palm region. This is followed by the selection of four reference points from the set of 12 obtained points. The midpoints of these points are computed, which means the midpoints contribute as the adjacent corner for the ROI. The 


\section{International Journal of Science and Research (IJSR) \\ ISSN (Online): 2319-7064}

Index Copernicus Value (2013): 6.14 | Impact Factor (2014): 5.611

GIST features of the ROIs are extracted by the GIST descriptor. The extracted features are then distinguished and matched by the ELM classifier.

\section{Experimental Analysis}

To the best of our knowledge, at present the only publicly available high-resolution palmprint database is the Tsinghua Palmprint Database (THUPALMLAB) [8], [36]. It contains 1280 palmprint images from 80 different subjects (left and right palms of each subject, eight impressions per palm). All the images were captured using a palmprint scanner from Hisign; the image size is $2040 \times 2040$ pixels, the resolution $500 \mathrm{dpi}$, and the images have 256 gray levels. The THUPALMLAB database consists of two separate sets: 1) training set and 2) test set. The former contains 320 palmprints (from 40 different palms), while the latter contains the remaining 960 palmprints (from 120 different palms). The proposed work is analysed with respect to the recognition accuracy and time consumption.

The recognition accuracy of any palmprint recognition system must be greater such that the system perfectly recognises the palmprints. The time consumption of the proposed work must be as lesser as possible. The proposed work satisfies both these statements and the results are given below.

Table 1: Performance analysis

\begin{tabular}{|c|c|c|}
\hline Method & Recognition Rate & $\begin{array}{c}\text { Time consumption } \\
\text { (seconds) }\end{array}$ \\
\hline Jain and Feng (2009) & $82 \%$ & 58 \\
\hline Dai and Zhou (2011) & $88 \%$ & 67 \\
\hline Proposed work & $98.8 \%$ & 5 \\
\hline
\end{tabular}

Thus, the proposed work meets the standard requirements of a palmprint recognition system. The results are compared with the existing works and our work outperforms the other works.

\section{Conclusion}

This paper presents a novel palmprint recognition system which is composed of three stages. The first stage is concerned with the process of pre-processing the palmprint images. Feature extraction is the second phase in which the fine features of the palmprints are extracted by the GIST descriptors. Finally, ELM is employed as the classifier to recognise between the palmprints. The experimental outcome of the proposed work is satisfactory.

\section{References}

[1] DUTA, N., JAIN, A., AND MARDIA, K. 2002. Matching of palmprints. Pattern Recog. Lett. 23, 4), 477-485.

[2] JAIN, A. K., ROSS, A., AND PRABHAKAR, S. 2004. An introduction to biometric recognition. IEEE Trans. Circuits Syst. Video Technol. 14, 1, 4-20.

[3] SHU, W. AND ZHANG, D. 1998. Automated personal identification by palmprint. Opt. Eng. 38, 8, 2359-2362.
[4] ZHANG, D. 2004. Palmprint Authentication. Kluwer Academic, USA.

[5] ZHANG, D. AND SHU, W. 1999. Two novel characteristic in palmprint verification: Datum point invariance and line feature matching. Pattern Recog. 32, 4, 691-702.

[6] JAIN, A. AND FENG, J. 2009. Latent palmprint matching. IEEE Trans. Pattern Anal. Mach. Intell. 31, 5, 1032-1047.

[7] JAIN, A., CHEN, Y., AND DEMIRKUS,M. 2007. Pores and ridges: High-resolution fingerprint matching using level 3 features. IEEE Trans. Pattern Anal. Mach. Intell. $29,1,15-27$.

[8] JAIN, A. AND DEMIRKUS, M. 2008. On latent palmprint matching. technical report.

[9] LI, W., ZHANG, D., AND XU, Z. 2002. Palmprint identification by fourier transform. Int. J. Pattern Recog. Artif. Intell. 16, 4, 417-432.

[10]ZHANG, D., KONG, W. K., YOU, J., AND WONG, M. 2003. Online palmprint identification. IEEE Trans. Pattern Anal. Mach. Intell. 25, 9, 1041-1050.

[11] J. Dai and J. Zhou, "Multi-feature based high-resolution palmprint recognition," IEEE Trans. Pattern Anal. Mach. Intell., vol. 33, no. 5, pp. 945-957, May 2011.

[12] Tsinghua Univ. Dept. Autom., i-Vision Group Web Site, 2011. [Online]. Available: http://ivg.au.tsinghua.edu.cn

\section{Author Profile}

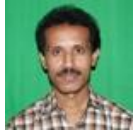

S. Chidambaranathan, received his post graduate degree in Mathematics from Madurai Kamaraj University, Madurai. He also earned post graduate degree in Computer Application and doctorate in computer Science from Manonmaniam Sundaranar University, Tirunelveli. Presently he is working as Head of the Department of MCA at St. Xavier's College (Autonomous), Palayamkottai, TamilNadu. He is an author for many books including "PHP for beginners", "XML- An Practical approach" and "Everything HTML". He has published many research papers in National, International journals and conference proceedings. 